\title{
An Objective-Prism Survey of Emission-Line Galaxies
}

\author{
Chulhee Kim \\ School of Physics and Technology, Chonbuk National University, Korea
}

\begin{abstract}
In order to discover emission-line galaxy (ELG) candidates towards the Hydra Void, an objective-prism survey was undertaken. As a first step, five fields were observed with the UK Schmidt Telescope, and we discovered a total of 33 candidates on a single prism plate through the $[\mathrm{O} \mathrm{HI}] \lambda \lambda 4959,5007$ emission feature.
\end{abstract}

\section{Introduction}

During last two decades, a number of major surveys for blue emission-line galaxies have been carried out, and a wide variety of types of objects have been discovered. However, the properties of galaxies inhabiting voids are not well studied, both because of their small numbers and the difficulty of obtaining complete, unbiased samples of these objects. Voids present the opposite environmental conditions from clusters, and galaxies in voids can reveal how galaxies evolve in a relatively undisturbed environment. Investigations into the nature and distribution of galaxies in voids may also provide clues to the physical properties of low-density regions.

The discovery of a large volume in Boötes that contains no known normal galaxies, but does contain nine or ten emission-line galaxies (Moody et al. 1987), raises questions about the connection between galaxy population density and individual galaxy properties in places far from rich clusters. Interestingly, no galaxy has been found in the Coma Void (Gregory et al. 1988). Also, these emission-line galaxies are not dwarf galaxies, unlike the predictions of theoretical models of galaxy formation in voids (e.g., Dekel \& Silk 1986). There is also evidence that galaxies away from clusters have a higher frequency of line emission than rich-cluster members by a factor of about 3 or 4 . Understanding the nature of emission-line galaxies is, therefore, very important in the construction of a global picture of galaxy and large-scale structure.

\section{Observations}

Among the various available survey techniques, we adopted the objective-prism survey to maintain consistency and to enable a comparison between our survey results and those for northern voids. As a first step, we observed five fields towards the Hydra Void with the UK Schmidt Telescope during the spring of 1994. The prism plates used for the search were taken with the $2^{\circ}$ prism and covered the range $4550-6900 \AA$. This gives a dispersion of $830 \AA \mathrm{mm}^{-1}$ at $\mathrm{H} \gamma$ 
and a resolution of $18 \AA$. The limiting magnitude reaches about $18.0 \mathrm{mag}$ with a 20 min. exposure time.

Although usually IIIa-J or IIIa-F emulsions are widely used, we tried 4415 Tech Pan film. This film has proven to be extremely successful in a new survey of low surface-brightness galaxies (Phillipps \& Parker 1993). Scanning of the prism plates and direct $R$ plates was performed by the APM at the Royal Observatory.

\section{Discussion}

Usually Schmidt plates are automatically searched with effective software because there are more than ten thousand objects on a single plate. Instead, we searched by visual inspection because the observed fields are very crowded and the emission features are faint. In this case, emission features can be misidentified due to the duplication of spectra produced by neighboring objects.

Both images of a scanned prism plate and a direct $R$ plate were displayed on monitor simultaneously using the 'IMTOOL' package. Once the emission feature [O III] $\lambda \lambda 4959,5007$ is identified, the corresponding object on the direct $R$ plate was examined to see whether this emission feature is produced by a galaxy. Through this procedure, we discovered in total 33 candidates of ELGs on a single field. Now four other plates are under investigation and observations of other fields will be carried out to cover about total 20 fields of the entire Hydra Void.

\section{References}

Dekel, A., \& Silk, J. 1986, ApJ, 303, 39.

Gregory, S. A., Tifft, W. G., \& Moody, J.W. 1988, ApJ, 95, 662.

Moody, J. W., Kirshner, R. P., MacAlpine, G. M., \& Gregory, S. A. 1987, ApJL, $314, \mathrm{~L} 33$.

Phillipps, S., \& Parker, Q.A. 1993, MNRAS, 265, 385. 